\title{
Autologous monocyte-derived DC vaccination combined with cisplatin in stage III and IV melanoma patients: a prospective, randomized phase 2 trial
}

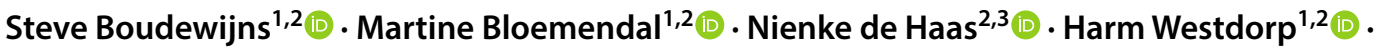

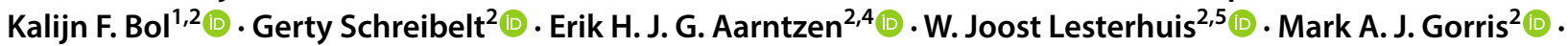 \\ Alexandra Croockewit ${ }^{6}$. Lieke L. van der Woude ${ }^{2,7} \cdot$ Michelle M. van Rossum $^{8} \cdot$ Marieke Welzen $^{3} \cdot$ Anna de Goede $^{3}$. \\ Stanleyson V. Hato ${ }^{2}$ - Winette T. A. van der Graaf ${ }^{1}$. Cornelis J. A. Punt ${ }^{9}$ (D) Rutger H. T. Koornstra ${ }^{1,10} \mathbb{D}$. \\ Winald R. Gerritsen ${ }^{1}$ (D) Carl G. Figdor ${ }^{2}$ (D) . I. Jolanda M. de Vries ${ }^{1,2}(\mathbb{D}$
}

Received: 12 July 2019 / Accepted: 28 December 2019 / Published online: 24 January 2020

(c) The Author(s) 2020

\begin{abstract}
Background Autologous dendritic cell (DC) vaccines can induce tumor-specific T cells, but their effect can be counteracted by immunosuppressive mechanisms. Cisplatin has shown immunomodulatory effects in vivo which may enhance efficacy of DC vaccination.

Methods This is a prospective, randomized, open-label phase 2 study (NCT02285413) including stage III and IV melanoma patients receiving 3 biweekly vaccinations of gp100 and tyrosinase mRNA-loaded monocyte-derived DCs with or without cisplatin. Primary objectives were to study immunogenicity and feasibility, and secondary objectives were to assess toxicity and survival.

Results Twenty-two stage III and 32 stage IV melanoma patients were analyzed. Antigen-specific CD8 ${ }^{+} \mathrm{T}$ cells were found in $44 \%$ versus $67 \%$ and functional T cell responses in $28 \%$ versus $19 \%$ of skin-test infiltrating lymphocytes in patients receiving DC vaccination with and without cisplatin, respectively. Four patients stopped cisplatin because of toxicity and continued DC monotherapy. No therapy-related grade 3 or 4 adverse events occurred due to DC monotherapy. During combination therapy, one therapy-related grade 3 adverse event, decompensated heart failure due to fluid overload, occurred. The clinical outcome parameters did not clearly suggest significant differences.

Conclusions Combination of DC vaccination and cisplatin in melanoma patients is feasible and safe, but does not seem to result in more tumor-specific $\mathrm{T}$ cell responses or improved clinical outcome, when compared to DC vaccination monotherapy.
\end{abstract}

Keywords Dendritic cell $\cdot$ Vaccination $\cdot$ Cisplatin $\cdot$ Melanoma $\cdot$ Immunotherapy

Steve Boudewijns, Martine Bloemendal and Nienke de Haas have contributed equally.

Note on previous publication: Parts of this publication were published before in the doctoral thesis 'Dendritic cell vaccination in the evolving therapeutic landscape of melanoma' by S. Boudewijns in 2017 and in the doctoral thesis 'Novel strategies in dendritic-cell based immunotherapy-Focusing on adjuvant treatment of stage III melanoma' by M. Bloemendal in 2019 [ 1 , 2]. Both were written at the departments of Tumor Immunology and Medical Oncology of the Radboud university medical center, Nijmegen, the Netherlands.

Electronic supplementary material The online version of this article (https://doi.org/10.1007/s00262-019-02466-x) contains supplementary material, which is available to authorized users.

Extended author information available on the last page of the article

$\begin{array}{ll}\text { Abbreviations } \\ \text { AJCC } & \text { American Joint Cancer on Committee } \\ \text { CBA } & \text { Cytometric bead array } \\ \text { CTCAE } & \text { Common terminology criteria for adverse } \\ & \text { events } \\ \text { DTH } & \text { Delayed-type hypersensitivity } \\ \text { EBV } & \text { Epstein-Barr virus } \\ \text { FFPE } & \text { Formalin-fixed paraffin embedded } \\ \text { HS } & \text { Human serum } \\ \text { ICI } & \text { Immune checkpoint inhibitors } \\ \text { KLH } & \text { Keyhole limpet hemocyanin } \\ \text { M-MDSC(s) } & \text { Monocytic myeloid-derived suppressor } \\ & \text { cell(s) } \\ \text { mIHC } & \text { Multiplex immunohistochemistry } \\ \text { PD-L2 } & \text { Programmed death ligand 2 }\end{array}$




$\begin{array}{ll}\text { pSTAT } & \begin{array}{l}\text { Phosphorylated signal transducer and acti- } \\ \text { vator of transcription }\end{array} \\ \text { RFS } & \text { Recurrence-free survival } \\ \text { SKIL(s) } & \text { Skin-test infiltrating lymphocyte(s) } \\ \text { TM } & \text { Tetramer }\end{array}$

\section{Introduction}

Based on their capacity to activate and prime naïve $\mathrm{T}$ cells, dendritic cells (DCs) are the most efficient antigen-presenting cells of the immune system. This makes them ideal candidates to be exploited for vaccination therapies [3]. Studies with autologous DC vaccines have shown to induce tumorspecific immune responses in both lymph node involved (stage III) and metastatic (stage IV) melanoma patients [4]. Despite immunological responses, objective clinical responses are rare in stage IV melanoma patients [5-7]. In stage III patients, a higher percentage of immunological responses to DC vaccination are observed, which could be explained by a lower tumor burden and concomitant less tumor-induced immune suppression. Accordingly, we found favorable overall survival (OS) in stage III melanoma patients who received adjuvant DC vaccination compared to matched controls [8], which is currently investigated in our randomized phase 3 trial (NCT02993315).

Combination with therapies modulating an immunosuppressive tumor microenvironment (TME) may strengthen the effect of DC vaccination. Platinum-based chemotherapeutics are widely used for several types of cancer [9]. Cisplatin was also tested in metastatic melanoma patients, as monotherapy and in combination with other types of chemotherapy, interferon (IFN) or interleukin (IL)-2. However, without great clinical benefit and with more toxicity than dacarbazine, another chemotherapeutic, dacarbazine was the preferred systemic therapy at time of trial enrollment [10-13]. The rationale to combine DC vaccination with cisplatin is based on the ability of cisplatin to not only cross-link DNA and inhibit mitosis, but also to have immunomodulatory effects $[14,15]$. In vitro platinum drugs cause inhibition of signal transducer and activator of transcription (STAT) signaling via decreasing phosphorylation of different STAT proteins [16]. STAT proteins each have a different effect on the anti-tumor response [17, 18]. For example, diminished STAT6 phosphorylation results in downregulation of the T cell inhibitory molecule programmed death ligand 2 (PD-L2) on both DCs and tumor cells, which enhances tumor cell recognition by $\mathrm{T}$ cells $[15,18]$. More recently, preclinical studies showed that cisplatin may upregulate MHC class I expression on tumor cells and upregulate the lytic activity of cytotoxic effector cells [14]. Furthermore, it has been shown that cisplatin can improve recruitment of immune effector cells to the
TME and enhances their proliferation and at the same time causes downregulation of immunosuppressive cells in the TME by reducing levels of myeloid-derived suppressor cells (MDSCs) and regulatory T cells (Tregs) [14, 19]. Besides this effect in the TME, a reduction in circulating Tregs was found in patients with non-small cell lung cancer after treatment with cisplatin and vinorelbine [20]. Finally, it was recently observed that cisplatin can induce immunogenic cell death [21].

In a preclinical tumor model, synergy was shown between vaccination with synthetic long peptides of human papillomavirus (HPV) type 16 and cisplatin. Combined treatment led to highly infiltrated tumors with HPV-specific tumor necrosis factor (TNF)- $\alpha$ and IFN- $\gamma$ producing T cells and significantly decreased tumor cell proliferation compared to single treatment [22]. Combining DC vaccination with cisplatin may have a similar synergistic effect, as the immunomodulatory effects of cisplatin potentially improve the efficacy of the antigen-specific $\mathrm{T}$ cells induced by $\mathrm{DC}$ vaccination.

The aim of this study was to explore whether the combination of autologous DC vaccination and cisplatin in stage III and IV melanoma patients is feasible and safe and whether it leads to better immunological and clinical responses compared to DC monotherapy.

\section{Materials and methods}

\section{Patient characteristics}

Patients between 18 and 70 years of age with histologically confirmed stage III or IV melanoma, both with a cutaneous (American Joint Cancer on Committee (AJCC) 7th edition [23]) and uveal (AJCC 7th edition [24]) melanoma, were eligible. Additional key eligibility criteria included: WHO performance status of 0 or 1 ; melanoma expressing gp100 (compulsory) and tyrosinase (non-compulsory) as assessed by immunohistochemistry performed on previously obtained tissue; normal serum lactate dehydrogenase (LDH); life expectancy of at least 3 months; serum creatinine level $<150 \mu \mathrm{mol} / \mathrm{L}$; within 2 months of radical lymph node dissection; and at least one measurable target lesion according to Response Evaluation Criteria in Solid Tumors (RECIST) version 1.1 in stage IV patients. Key exclusion criteria were: any prior chemotherapy, immunotherapy or radiotherapy within 4 weeks of the first vaccination; symptomatic brain metastases; rapidly progressive symptomatic disease; a history of any second malignancy in the previous 5 years; autoimmune disease; use of immunosuppressive drugs; and a known allergy to shell fish due to the use of Keyhole limpet hemocyanin (KLH) protein. 


\section{Study design and treatment}

In this open-label phase 2 study, patients were randomly assigned between two experimental arms in a 1:1 ratio to receive autologous DC vaccination with or without cisplatin, stratified for disease stage (III versus IV). Unresectable stage III was considered stage IV disease. Vaccines consisted of autologous cytokine-matured monocyte-derived DCs electroporated with mRNA encoding gp100 and tyrosinase. Patients received three biweekly vaccinations, followed by a delayed-type hypersensitivity (DTH) skin test (Supplementary Fig. 1). Patients received two additional cycles of vaccinations at 6 -month intervals. Cisplatin $\left(50 \mathrm{mg} / \mathrm{m}^{2}\right.$, maximum of $100 \mathrm{mg} / \mathrm{dose}$ ) was administered intravenously 1-2 $\mathrm{h}$ before DC injection. The dose was based on in vitro experiments, evaluating the effect on cytokine production of DCs [18]. As cisplatin is highly emetogenic, the standard antiemetic regime consisted of dexamethasone $(10 \mathrm{mg}$ intravenously on day 1 or $12 \mathrm{mg}$ orally on day $1-4$ ), aprepitant, ondansetron and metoclopramide. Since February 2014, this regime changed to $12 \mathrm{mg}$ orally on day $1-4$. Primary objectives of the study were to study immunological response and feasibility of the addition of cisplatin to DC vaccination. Therefore, patients were replaced when no DTH skin test was performed. Secondary objectives were to assess toxicity, recurrence-free survival (RFS), progression-free survival (PFS) and OS. Toxicity was assessed according to the National Cancer Institute Common Terminology Criteria for Adverse Events (CTCAE) version 3.0. Tumor evaluation was performed at baseline and every 3 months thereafter by physical examination in stage III patients and by a CT scan of chest and abdomen evaluated according to RECIST version 1.1 in stage IV patients. Treatment was stopped with disease recurrence (stage III), progression (stage IV), unacceptable toxicity or withdrawal of consent.

\section{Vaccine production}

Monocytes were enriched from leukapheresis products as described before [25]. Monocytes were cultured in X-VIVO 15 medium (Lonza) supplemented with $2 \%$ human serum (HS; Sanquin), IL-4 (500 U/ml), GM-CSF ( $800 \mathrm{U} / \mathrm{ml}$, both CellGenix) and KLH (10 $\mu \mathrm{g} / \mathrm{ml}$, Calbiochem). DCs were matured with a cocktail of $10 \mathrm{ng} / \mathrm{ml} \mathrm{TNF-} \alpha, 5 \mathrm{ng} / \mathrm{ml} \mathrm{IL-} 1 \beta$, $15 \mathrm{ng} / \mathrm{ml}$ IL-6 (all CellGenix) and prostaglandin $\mathrm{E}_{2}(10 \mu \mathrm{g} /$ $\mathrm{ml}$, Pharmacia \& Upjohn) [26]. Cells used for the DTH skin test were cultured without KLH. DCs were electroporated with mRNA encoding gp100 or tyrosinase, as previously described [27]. Patients could only participate if the predefined phenotypic minimal release criteria used in clinical trials were met [28]. DCs were administered both intradermally (maximum of $10 \times 10^{6}$ cells) and intravenously (maximum of $20 \times 10^{6}$ cells).

\section{Flow cytometry}

Phenotype of the ex vivo generated DCs was characterized by flow cytometry with the following monoclonal antibodies (mAbs): anti-HLA-ABC-PE, anti-HLA-DR-PE, anti-CD80-PE, anti-CD83-PE, anti-CD86-PE, anti-CD3PE, anti-CD25-PE, anti-CD95-PE (all BD Biosciences), anti-CD14-PE (Sanquin Reagents), anti-HLA-DQ-PE, anti-CD20-PE (both BioLegend) and anti-CCR7-PE (Miltenyi Biotec). For intracellular staining, anti-gp100 (NKI/beteb; Netherlands Cancer Institute) and anti-tyrosinase (T311; Cell Marque Corp) were used. Flow cytometry was carried out using a FACSCalibur flow cytometer equipped with CellQuest software (BD Biosciences).

The presence of Tregs and monocytic (M)-MDSCs was analyzed in PBMCs isolated from heparinized blood collected prior to the apheresis and on the day of and prior to the first DTH skin test by Ficoll-Paque density centrifugation. The Treg antibody panel consisted of fixable viability dye 780, anti-FoxP3-Alexa488 (both eBioscience), anti-CD3-BV605 (BioLegend), anti-CD4BV510 and anti-CD25-BV421 (both BD Biosciences). M-MDSCs were analyzed with anti-CD33-APC (BioLegend), anti-CD14-BV421, anti-HLA-DR-BV510 and antiCD11b-BV605 (all BD Biosciences) antibodies. Flow cytometry was carried out using a FACSLyric equipped with FACSuite software (BD Biosciences). Tregs were identified as $\mathrm{CD}^{+}{ }^{+} \mathrm{CD} 4^{+} \mathrm{CD} 25^{+} \mathrm{FoxP} 3^{+}$cells. M-MDSCs were analyzed as $\mathrm{HLA}-\mathrm{DR}^{-} \mathrm{CD} 14^{+} \mathrm{CD} 11 \mathrm{~b}^{+} \mathrm{CD} 33^{+}$cells. Analyses were carried out using FlowJo software version 10.0.7 (Treestar Inc.).

\section{KLH-specific proliferation}

PBMCs were stimulated with KLH $\left(4 \mu \mathrm{g} / 2 \times 10^{5}\right.$ PBMC; Immucothel, Biosyn) in X-VIVO 15 with $2 \%$ HS. After 3 days, cells were incubated with ${ }^{3} \mathrm{H}$-thymidine for $8 \mathrm{~h}$ and incorporation was measured with a $\beta$-counter. Experiments were performed in sextuplicate, and ovalbumin was used as control. Response to KLH is given as proliferation index (proliferation with KLH/proliferation without KLH).

\section{Skin-test infiltrating lymphocyte culture analysis}

One to two weeks after each vaccination cycle, skin tests were performed, as described previously [29]. In short, DCs electroporated with gp100 and/or tyrosinase $\left(1 \times 10^{6}\right.$ DCs maximum in total) were thawed and injected intradermally at the back of patients at four different sites. After 2 days, punch biopsies $(6 \mathrm{~mm})$ were taken. Half of each biopsy was manually cut and cultured for 2-4 weeks 
in RPMI-1640 containing 7\% HS and IL-2 (100 U/ml, Novartis).

Skin-test infiltrating lymphocyte (SKIL) cultures and PBMCs from HLA-A2.1 positive patients were stained with HLA-A2.1 tetrameric MHC complexes containing the epitopes gp100:154-162, gp100:280-288 or tyrosinase:369-377 (Sanquin) as described before [30]. Human immunodeficiency virus was used as negative control. Tetramer positivity $\left(\mathrm{TM}^{+}\right)$was defined as at least a twofold increase in the double positive population compared to control. In HLA-A2.1 positive patients, the production of IFN- $\gamma$ was measured in supernatants after $16 \mathrm{~h}$ of co-culture with different target cells: T2 cells pulsed with gp100:154-162, gp100:280-288 or tyrosinase:369-377; BLM (HLA-A2.1positive melanoma cell line without endogenous expression of gp100 and tyrosinase) transfected with gp100, tyrosinase or control antigen G250; and Mel624 (HLA-A2.1positive, gp100-positive and tyrosinase-positive tumor cell line). Cytokine analysis was performed by cytometric bead array (CBA) (human Th1/Th2 FlowCytomix multiplex kit, eBioscience).

In HLA-A2.1-negative patients, cytokine production by SKILs was determined by using autologous Epstein-Barr virus (EBV)-transformed B cells as described by van Nuffel et al. [31]. Autologous EBV-B cells were generated from PBMCs and electroporated with mRNA encoding full-length gp100 or tyrosinase (Curevac $\mathrm{GmbH}$ ). Carcinoembryonic antigen (CEA) was used as a negative control. MRNAloaded EBV-B cells were co-cultured 1:1 with SKILs for $24 \mathrm{~h}$. Afterward, expression of the early activation markers CD69, CD107a and CD137 on CD8 ${ }^{+} \mathrm{T}$ cells was analyzed. Phorbol myristate acetate-stimulated $(5 \mu \mathrm{g} / \mathrm{ml}$; SigmaAldrich) SKILs were used as positive control. After $24 \mathrm{~h}$ of co-culture, cytokine production was measured with CBA.

\section{Multiplex immunofluorescence staining}

Tumor tissue resected prior to the start and after experimental therapy was collected if available. Sections of $4 \mu \mathrm{m}$ from formalin-fixed paraffin-embedded (FFPE) tissue were deparaffinized, and sections from frozen tissue were dried and fixed in $4 \%$ formaldehyde.

Three-color multiplex immunohistochemistry (mIHC) using Opal 7-Color IHC Kit (NEL801001KT, Perkin Elmer) was performed for the detection of STAT3 and phosphorylated STAT3 (pSTAT3). After antigen retrieval, tissue sections were subjected to mAbs listed in Supplementary Table 1a.

A seven-color mIHC for the detection of lymphocyte populations was applied using the BOND RX IHC \& ISH Research Platform (Leica Biosystems) with mAbs for CD45RO, CD8, CD20, CD3, Foxp3 and a melanoma mix as listed in Supplementary Table 1b. For analysis,
$\mathrm{CD} 3^{+} \mathrm{CD} 8^{-}$cells were considered $\mathrm{CD} 4^{+} \mathrm{T}$ cells. All epitope retrievals and antibody-TSA complex removals were performed using BOND Epitope Retrieval 2 (AR9640, Leica Biosystems). Blocking steps were performed with antibody diluent for $10 \mathrm{~min}$, primary antibody incubations for $1 \mathrm{~h}$, secondary antibody Opal polymer HRP Ms + Rb incubations for $30 \mathrm{~min}$ and Opal reagent incubations for $10 \mathrm{~min}$, all at room temperature. Tissue was counterstained with 4',6-diamidino2-phenylindole (DAPI) and mounted with Fluoromount-G (0100-01; Southern Biotech). A similar seven-color mIHC was performed on cryopreserved tissue with anti-granzyme $\mathrm{B}$ instead of CD20 (Supplementary Table 1c).

The slides were scanned using the Automated Quantitative Pathology Imaging System Vectra 3.0.4. Regions of interest were selected using Phenochart version 1.0.9 for multispectral imaging at $20 \times$ magnification. InForm version 2.2.1. was used for spectral unmixing of Opal fluorophores, DAPI and autofluorescence and downstream imaging analysis (all PerkinElmer Inc.).

For pSTAT3 analysis, the percentage of nuclei containing pSTAT3 was counted separately by two investigators. Divergent results were discussed to reach consensus. For lymphocyte analysis, a selection of 30-35 representative original multispectral images was used to train InForm to distinguish tumoral from stromal tissue and background based on DAPI and autofluorescence. Settings for adaptive cell segmentation were based on DAPI and membrane signals. All settings applied to the training images were saved in an algorithm to allow batch analysis. Segmented cell data were analyzed using FlowJo in which immune cells were phenotyped by manual gating and divided by the surface area of the tissue region $\left(\mathrm{mm}^{2}\right)$.

\section{Statistical analysis}

A sample size of 27 patients per arm was calculated using a two-sided log-rank test, to have $80 \%$ power with an alpha of 0.05 to detect an anticipated improvement from 30 to $70 \%$ in immunological response rate with the addition of cisplatin to DC vaccination. Survival was calculated from the date of apheresis to the first date of progression (PFS) in stage IV and recurrence (RFS) in stage III, using the Kaplan-Meier method. Difference between treatment groups was evaluated using a log-rank test. Recurrence and progression were censured in case of non-melanoma-related death. Follow-up duration was determined from date of apheresis to date of the last follow-up and censored for death. Immunological results after the first cycle were used to prevent a guarantee time bias. Paired $t$ tests were performed to evaluate KLH responses before and after vaccination and independentsamples $t$ tests to evaluate differences in KLH proliferation between groups. For $\mathrm{TM}^{+} \mathrm{CD}^{+} \mathrm{T}$ cells and functional $\mathrm{T}$ cells, differences between groups were evaluated using 
a Chi-square test or 2-sided Fisher's exact test in case of expected count $<5$. $p$ values $<0.05$ were considered significant. IBM SPSS Statistics version 25.0 (IBM Corp.) and Graphpad Prism 5.03 (GraphPad Software Inc.) were used for statistical analysis and data visualization.

\section{Results}

\section{Patient and vaccine characteristics}

Between February 2011 and July 2014, sixty patients were screened and included in the trial (Supplementary Fig. 2) of whom six were replaced: two stage IV patients because no acceptable DC product could be produced and four stage IV patients since they had progressive disease prior to the first immunological assessment. Therefore, 54 patients were included in the final analysis: 22 stage III and 32 stage IV melanoma patients. Patients were randomly assigned to receive either DC vaccination alone or combined with cisplatin. In all but one patient included, a DC product meeting the predefined minimal release criteria could be produced from the first apheresis (Supplementary Fig. 3a). In this particular patient, this was achieved after a repeated apheresis. Flow cytometry confirmed intracellular protein expression of both gp100 and tyrosinase in DCs (Supplementary Fig. 3b). In two patients, yield was insufficient for three vaccinations; therefore, apheresis was repeated during the first cycle.

Baseline characteristics of immunologically evaluable patients are summarized in Table 1. Overall, in the stage III group, five patients (23\%) had stage IIIA, 5 (23\%) had stage IIIB, and 11 (50\%) had stage IIIC disease. Most patients (73\%) with IIIC melanoma were randomized to receive monotherapy. Eleven patients $(50 \%)$ completed all three cycles of three vaccinations, seven patients receiving combination therapy and four patients with DC monotherapy.

The stage IV group included 26 patients $(81 \%)$ with metastatic cutaneous melanoma, $2(6 \%)$ with irresectable stage III disease and $4(13 \%)$ with metastatic uveal melanoma. Only five patients (16\%) completed two cycles, and thereof, $2(6 \%)$ completed the total of three cycles of vaccinations. One patient in the combination group with stable disease at the first evaluation scan at 3 months was referred for palliative surgical resection of a stable ileal metastasis to lower the risk of a gastrointestinal bleeding. At clinical data cutoff April 23, 2019, median follow-up was 62.3 months in stage III and 64.9 months in stage IV patients.

\section{Adverse events}

All evaluable patients $(n=54)$ were included in the safety analysis (Table 2). The remaining four patients who did not complete at least one cycle showed no striking features in their toxicity profile. In the combination group, frequent adverse events included nausea and fatigue. One treatmentrelated grade 3 adverse event occurred, consisting of decompensated heart failure due to fluid overload. Cisplatin was stopped because of adverse events in four patients (15\%) based on decompensated heart failure, a deep venous thrombosis, persistent grade 2 tinnitus and grade 2 nausea and fatigue. DC vaccination was continued as monotherapy in all four patients. The dose of cisplatin was reduced in one patient after vaccination five because of grade 2 nausea. Treatment-related adverse events leading to dose interruptions occurred in two patients treated with cisplatin; due to grade 2 tinnitus or grade 2 thrombocytopenia. In the group treated with DC monotherapy, the most frequent adverse events were flu-like symptoms usually lasting less than $48 \mathrm{~h}$ and injection site reactions. No grade 3-4 events were observed.

\section{Induction of de novo immune responses}

DCs were loaded with the control antigen KLH, to test the capability to induce de novo immune responses. PBMCs after consecutive vaccinations of the first cycle showed an increase in KLH-specific T cell proliferation compared to baseline in all patients without significant difference in mean increase between both treatment groups ( $p=0.453$; Fig. 1a). In addition, no significant difference in mean increase was seen between stage III and IV patients (data not shown).

\section{Induction of tumor antigen-specific T cells}

The presence of gp100- and tyrosinase-specific $\mathrm{CD} 8^{+} \mathrm{T}$ cells was tested with HLA-A2.1 tetrameric MHC-peptide complexes in both PBMCs and T cells cultured from biopsies of DTH injection sites (SKILs) of HLA -A2.1 positive patients. Eighteen HLA-A2.1-positive patients received combination therapy and 12 HLA-A2.1-positive patients DC monotherapy. $\mathrm{TM}^{+} \mathrm{CD}^{+} \mathrm{T}$ cells were found in PBMCs of two patients, one in each treatment group (Fig. 1b).

$\mathrm{TM}^{+} \mathrm{CD}^{+} \mathrm{T}$ cells were found more frequently in SKILs, in 16 out of 30 patients (53\%) (Fig. 1c). There was no difference between treatment groups: $44 \%$ in the combination and $67 \%$ in the monotherapy group. In stage III patients, $33 \%$ treated with combination therapy compared to $86 \%$ treated with DC monotherapy showed $\mathrm{TM}^{+} \mathrm{CD} 8^{+}$SKILs. In stage IV patients, $56 \%$ of patients in the combination group compared to $40 \%$ in the monotherapy group showed $\mathrm{TM}^{+} \mathrm{CD}^{+}$ SKILs. Regardless of the treatment arm, $\mathrm{TM}^{+} \mathrm{CD}^{+}$SKILs were found in $56 \%$ of stage III and $50 \%$ of stage IV patients.

SKILs were analyzed for the occurrence of a functional $\mathrm{T}$ cell response, by measuring production of IFN $\gamma$ in response to cells loaded with gp100 or tyrosinase. This was found 
Table 1 Baseline characteristics

\begin{tabular}{|c|c|c|c|c|}
\hline & \multicolumn{2}{|c|}{ Stage III melanoma patients } & \multicolumn{2}{|c|}{ Stage IV melanoma patients } \\
\hline & $\begin{array}{l}\text { DC vaccina- } \\
\text { tion }(n=11)\end{array}$ & $\begin{array}{l}\text { DC vaccina- } \\
\text { tion + cisplatin } \\
(n=11)\end{array}$ & $\begin{array}{l}\text { DC vaccina- } \\
\text { tion }(n=16)\end{array}$ & $\begin{array}{l}\text { DC vaccina- } \\
\text { tion + cisplatin } \\
(n=16)\end{array}$ \\
\hline \multicolumn{5}{|l|}{ Sex, $n(\%)$} \\
\hline Male & $9(82)$ & $9(82)$ & $8(50)$ & $10(63)$ \\
\hline Female & $2(18)$ & $2(18)$ & $8(50)$ & $6(38)$ \\
\hline Age (years)—median (range) & $53(25-69)$ & $48(25-67)$ & $61(34-69)$ & $54(30-69)$ \\
\hline \multicolumn{5}{|l|}{ HLA-A2.1, $n(\%)$} \\
\hline Positive & $7(64)$ & $9(82)$ & $5(31)$ & $9(56)$ \\
\hline Negative & $4(36)$ & $2(18)$ & $11(69)$ & $7(44)$ \\
\hline \multicolumn{5}{|c|}{ Site of primary melanoma, $n(\%)$} \\
\hline Skin & $10(91)$ & $10(91)$ & $12(75)$ & $12(75)$ \\
\hline Eye & $0(0)$ & $0(0)$ & $3(19)$ & $1(6)$ \\
\hline Unknown primary & $1(9)$ & $0(0)$ & $1(6)$ & $3(19)$ \\
\hline Primary not assessed & $0(0)$ & $1(9)$ & $0(0)$ & $0(0)$ \\
\hline \multicolumn{5}{|l|}{ AJCC stage (7th edition) $)^{\mathrm{a}}, n(\%)$} \\
\hline IIIA & $2(18)$ & $3(27)$ & n.a & n.a \\
\hline IIIB & $1(9)$ & $4(36)$ & & \\
\hline IIIC & $8(73)$ & $3(27)$ & & \\
\hline IIIX & $0(0)$ & $1(9)$ & & \\
\hline \multicolumn{5}{|l|}{ Adjuvant radiotherapy, $n(\%)$} \\
\hline No & $7(64)$ & $8(73)$ & n.a & n.a \\
\hline Yes & $4(36)$ & $3(27)$ & & \\
\hline \multicolumn{5}{|l|}{ M stage at inclusion, $n(\%)$} \\
\hline M0 & n.a & n.a & $1(6)$ & $1(6)$ \\
\hline M1a & & & $3(19)$ & $4(25)$ \\
\hline M1b & & & $5(31)$ & $4(25)$ \\
\hline M1c & & & $7(44)$ & $7(44)$ \\
\hline \multicolumn{5}{|c|}{ Prior treatment for stage IV disease, $n(\%)$} \\
\hline No & n.a & n.a & $7(44)$ & $12(75)$ \\
\hline Surgery & & & $8(50)$ & $3(19)$ \\
\hline Radiotherapy & & & $1(6)$ & $0(0)$ \\
\hline Targeted therapy & & & $1(6)$ & $0(0)$ \\
\hline Chemotherapy & & & $1(6)$ & $0(0)$ \\
\hline Regional perfusion & & & $0(0)$ & $2(13)$ \\
\hline
\end{tabular}

${ }^{\mathrm{a}}$ The appropriate American Joint Committee on Cancer (AJCC) TNM system was used for both cutaneous (7th edition [23]) and uveal (7th edition [24]) melanomas

in 12 out of 51 patients (24\%) tested (Fig. 1d), without difference between patients treated with combination therapy (28\%) or DC monotherapy (19\%).

\section{STAT expression and T cell infiltrate in tumor tissue}

Tumor samples prior to and after DC vaccination were compared. Samples were not derived from the same tumor site, but we only used tissue of the same organ of origin, to obtain the best possible comparability. There were no clear differences in expression of nuclear pSTAT3 changes prior to and after DC vaccination between patients treated with monotherapy compared to combination therapy (Supplementary Fig. 4a, b). Therefore, we investigated other possible effects of cisplatin, such as decreasing numbers of Tregs in the TME. With $\mathrm{mIHC}$, we investigated the presence of $\mathrm{CD}^{+}{ }^{+} \mathrm{T}$ cells, $\mathrm{CD} 4^{+} \mathrm{T}$ cells and FoxP3 ${ }^{+}$cells. In these samples, no differences were observed between patients treated with or without cisplatin (Supplementary Fig. 4c-e).

Of one patient with a partial response to combined treatment of DC vaccination with cisplatin, sequential in-transit metastases were investigated (Fig. 2). This patient did not show a functional $\mathrm{T}$ cell response in SKILs, but showed clear development of tumor necrosis and expanding $\mathrm{T}$ cell 
Table 2 Treatment-related adverse events

\begin{tabular}{|c|c|c|c|c|c|c|}
\hline & \multicolumn{6}{|c|}{ Number of events (\%) } \\
\hline & \multicolumn{2}{|c|}{ DC vaccination $(n=27)$} & \multicolumn{3}{|c|}{ DC vaccination + cisplatin $(n=27)$} & \multirow[t]{2}{*}{$p$ value } \\
\hline & Grade 1 & Grade 2 & Grade 1 & Grade 2 & Grade 3 & \\
\hline Any event & $22(81)$ & $2(7)$ & $18(67)$ & $7(26)$ & $1(4)$ & 0.159 \\
\hline Injection site reaction & $20(74)$ & $1(4)$ & $10(37)$ & 0 & 0 & 0.008 \\
\hline Flu-like symptoms & $19(70)$ & $3(11)$ & $17(63)$ & 0 & 0 & 0.092 \\
\hline Nausea & $4(15)$ & 0 & $15(56)$ & $3(11)$ & 0 & $<0.001$ \\
\hline Vomiting & $4(15)$ & 0 & $1(4)$ & $3(11)$ & 0 & 0.091 \\
\hline Creatinine increased & $2(7)$ & 0 & $3(11)$ & 0 & 0 & 0.639 \\
\hline Constipation & 0 & 0 & $7(26)$ & 0 & 0 & 0.005 \\
\hline Fatigue & 0 & 0 & $4(15)$ & $3(11)$ & 0 & 0.018 \\
\hline Tinnitus & 0 & 0 & $3(11)$ & $2(7)$ & 0 & 0.064 \\
\hline
\end{tabular}

Adverse events that occurred in at least $10 \%$ of patients and were classified as possibly, probably or definitely related to the treatment by the investigator are depicted a

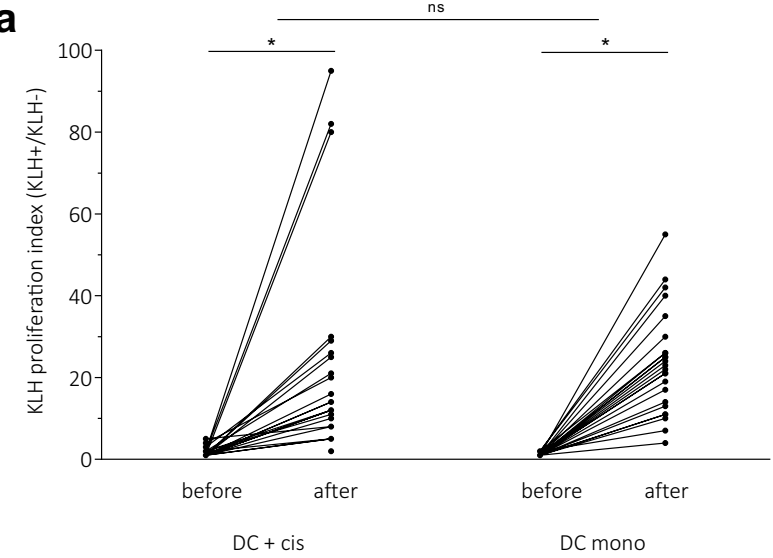

C

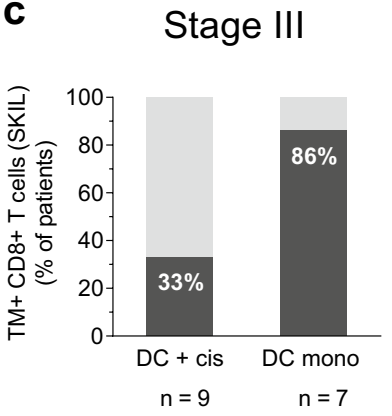

Stage IV

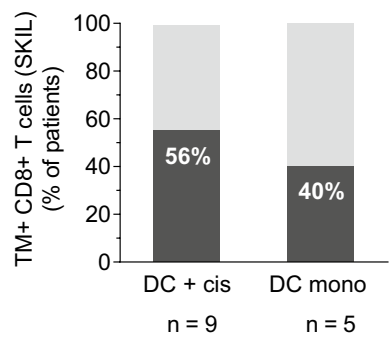

Fig. 1 Immunological responses. a KLH-specific T cell proliferation was measured before the start of therapy and after each vaccination of the first cycle in PBMCs of melanoma patients. The proliferative response to KLH is depicted as the highest proliferation index (proliferation with $\mathrm{KLH} /$ proliferation without $\mathrm{KLH}$ ) observed during the first cycle. b PBMCs were tested for $\mathrm{TM}^{+} \mathrm{CD}^{+} \mathrm{T}$ cells recog-

infiltration in a clinically responding metastasis during treatment.

Due to the retrospective nature of the tissue collection, only patients with recurrent or progressive disease

b
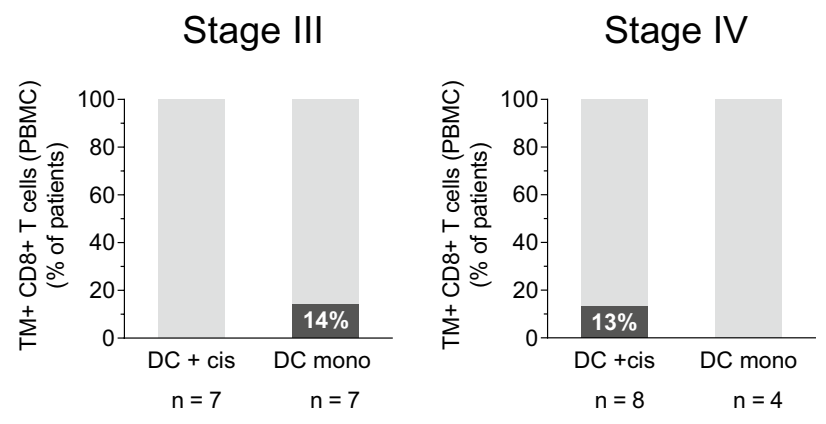

d
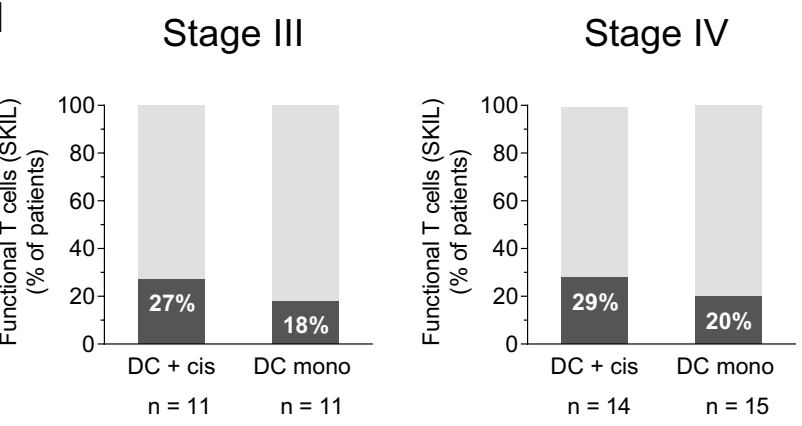

nizing gp100 or tyrosinase in HLA-A2.1 positive patients. c SKILs were tested for $\mathrm{TM}^{+} \mathrm{CD}^{+} \mathrm{T}$ cells recognizing gp100 or tyrosinase in HLA-A2.1 positive patients and $\mathbf{d}$ a functional $\mathrm{T}$ cell response in all patients. ${ }^{*} p<0.001, D C$ dendritic cell, $K L H$ Keyhole limpet hemocyanin, $n s$ not significant, $P B M C$ peripheral blood mononuclear cell, SKIL skin-test infiltrating lymphocytes, $T M$ tetramer

were included in the immunohistochemistry analysis. To address this caveat, we analyzed PBMCs for the presence of M-MDSCs and Tregs at baseline and after three vaccinations, as this was also available for patients 

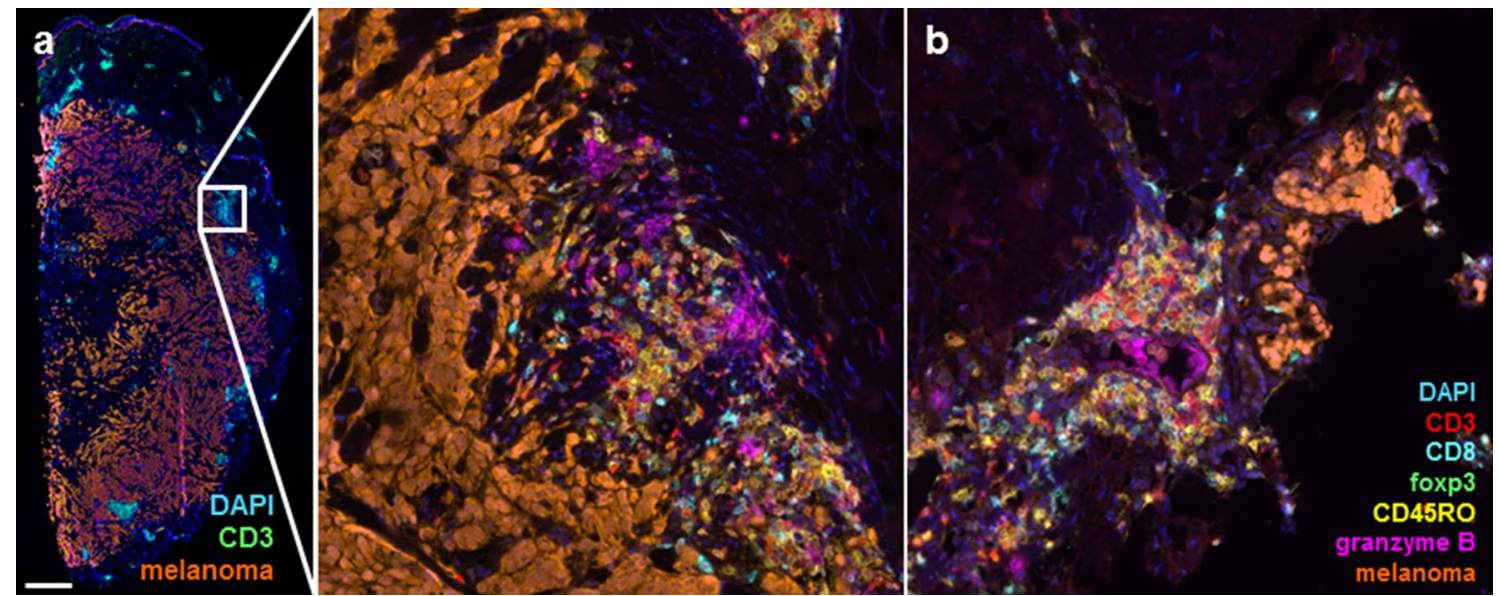

Fig. $2 \mathrm{~T}$ cell infiltration in melanoma metastases. Multispectral images of cutaneous metastases were taken at the start and during treatment in a patient with a partial response to dendritic cell vaccination in combination with cisplatin. a Metastasis at the start of treatment shows extensive melanoma cells with groups of $\mathrm{CD}^{+}$cells. When zoomed in (middle image), it reveals that $\mathrm{CD}^{+} \mathrm{T}$ cells and

without recurrent disease. No differences in the presence of M-MDSCs or Tregs between stage III melanoma patients treated with or without cisplatin were found (Supplementary Fig. 5).

\section{Clinical response}

Median RFS of stage III patients in the combination treatment group was 45.9 months versus 9.6 months in the monotherapy group ( $p=0.245$; Fig. $3 \mathrm{a}$ ). The median OS of stage III patients treated with cisplatin was not reached, as compared to 32.0 months without cisplatin ( $p=0.012$; Fig. $3 b$ ). One patient in the monotherapy group died because of a non-melanoma-related cause without evidence of recurrent disease.

Median PFS of stage IV patients in the combination group was 4.7 months, as compared to 3.0 months in the monotherapy group ( $p=0.101$; Fig. $3 c)$. When excluding metastatic uveal melanoma patients (one receiving cisplatin and three monotherapy), median PFS was 5.4 months in the combination group versus 3.5 months in the monotherapy group ( $p=0.121)$. Stage IV patients treated with monotherapy had a trend toward a longer OS with a median of 19.0 versus 12.2 months in the combination group $(p=0.063$; Fig. 3d). However, when excluding patients with uveal melanoma, this trend diminished. Subsequent treatment may have caused differences in OS, as more patients with progressive disease in the monotherapy than combination group received treatment with ipilimumab (69\% versus $31 \%$ ) or anti-PD1 antibodies (38\% versus $0 \%)$. granzyme B were present at the start of treatment. b Image of a clinically responding cutaneous metastasis after the second cycle of vaccinations combined with cisplatin, showing only few melanoma cells while an extensive $\mathrm{T}$ cell infiltrate was found, including $\mathrm{CD} 8^{+} \mathrm{T}$ cells and $\mathrm{CD}_{45 \mathrm{RO}^{+}}$cells

\section{Discussion}

In this randomized phase 2 trial, we showed that combining autologous DC vaccination with cisplatin is feasible and safe. Viable DCs, meeting the minimal release criteria [28], could be produced in $97 \%$ of patients. The toxicity profile of the combination treatment showed no unexpected safety concerns. Adverse events leading to discontinuation, interruption or dose reduction of cisplatin took place in a minority of patients. As expected, grade 1-2 injection site reactions and flu-like symptoms were common adverse events in both treatment groups, but occurred less in the combination group. These side effects might have been suppressed by dexamethasone, which was given as an antiemetic drug with cisplatin.

In this limited number of patients, the addition of cisplatin did not result in significantly improved immunological response to DC vaccination. In both treatment groups, DC vaccines induced de novo immune responses, despite the use of dexamethasone in the combination group. $\mathrm{TM}^{+} \mathrm{CD}^{+}$ SKILs were induced in about half of the patients without difference between the treatment groups, although in stage III melanoma patients, combination therapy might have induced less $\mathrm{TM}^{+} \mathrm{CD}^{+} \mathrm{T}$ cells than monotherapy.

The lack of improved immunological response with addition of cisplatin might be explained by several reasons. It is possible that the immunomodulatory effects of cisplatin in the dosage and regimen used are not strong enough to enhance the number of anti-tumor $\mathrm{T}$ cell responses in vivo significantly. Although we saw a clear increase in $\mathrm{T}$ cell infiltration in the metastasis of one responding patient to 
Stage III
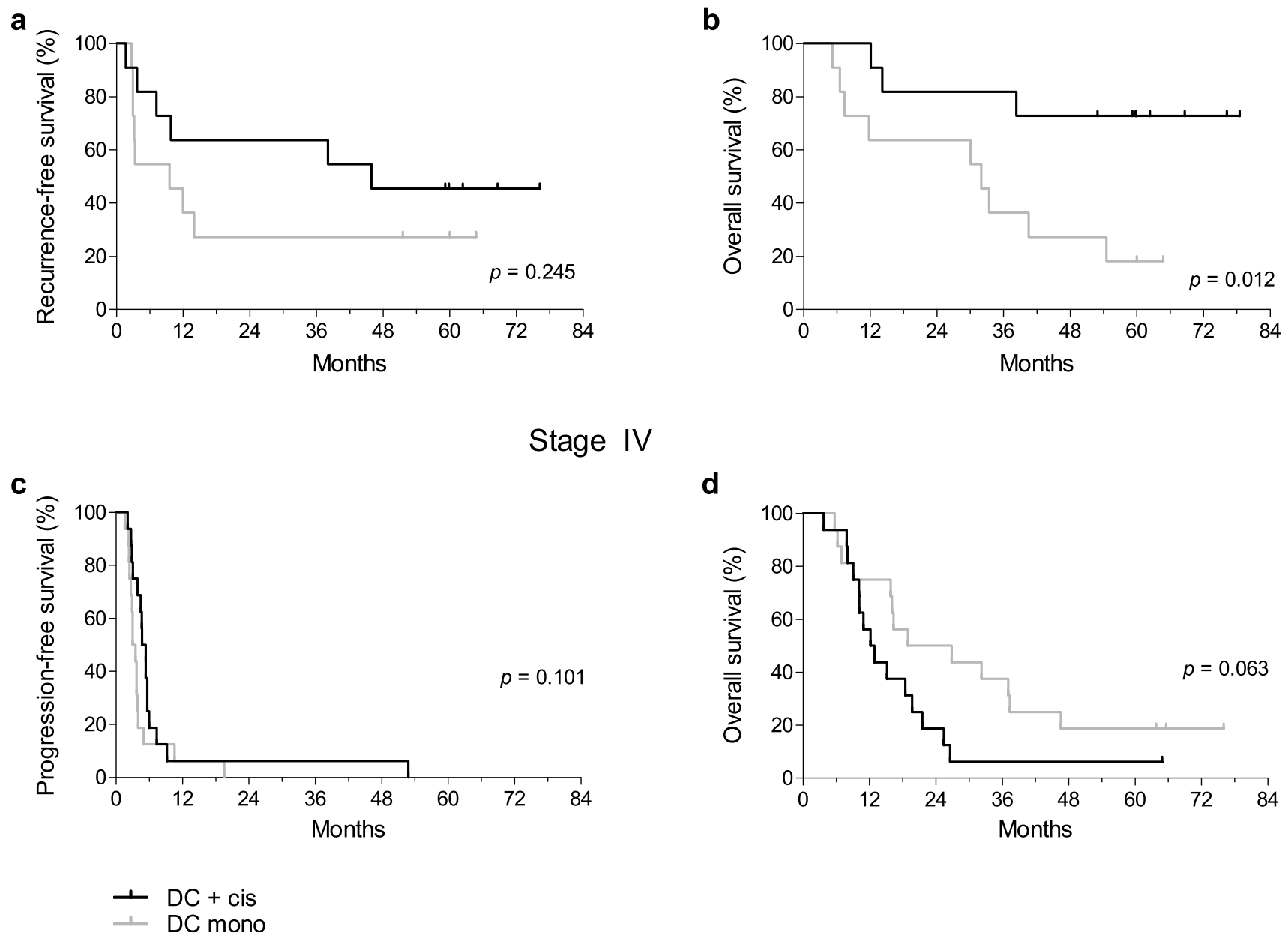

Fig. 3 Clinical responses in stage III and IV melanoma patients. Kaplan-Meier curves for a recurrence-free survival and $\mathbf{b}$ overall survival in stage III melanoma patients. For stage IV melanoma patients,

the combination treatment, we found little other evidence on the in vivo immunostimulatory effect of cisplatin. The timing of tumor sampling relative to the dosing of cisplatin might also be suboptimal, as the interval between the last dose of cisplatin and retrieval of tumor tissue was at least a few weeks. In previous studies, the in vitro effects on STAT expression were seen immediately after exposure to platinum drugs $[16,18]$. In addition, we investigated possible effects of cisplatin on the composition of the tumor immune infiltrate, showing no clear differences between treatment groups. Possibly explained by the retrospective nature of the collection of tumor material which retrieved only tissue of patients with progressive or recurrent disease. As pSTAT3 upregulation is associated with tumor proliferation, this is a probable explanation of the increased pSTAT3 expression after vaccination in all but two patients. Prospectively collected tumor biopsies are of interest for better comparability in both responders and non-responders.

Kaplan-Meier curves for $\mathbf{c}$ progression-free survival and $\mathbf{d}$ overall survival are shown

Dexamethasone could have had a negative effect on response induction in the combination group. Glucocorticosteroids can decrease the number of circulating T cells and increase the proliferation of Tregs [32, 33]. Therefore, dexamethasone might have hampered both the sensitivity of our immunomonitoring tests and the enhancing effects of cisplatin on the anti-tumor immune response. Also, the timing of the $\mathrm{DC}$ vaccination in relation to cisplatin might have caused the lack of synergy. In contrast to our study, Welters et al. found that carboplatin-paclitaxel every 3 weeks resulted in vigorous vaccine-induced $\mathrm{T}$ cell responses in advanced cervical cancer patients when a single dose of HPV16 synthetic long peptide vaccine was given 2 weeks after the second cycle of chemotherapy. This was despite use of $20 \mathrm{mg}$ dexamethasone intravenously as premedication. Their study showed that the decrease in circulating myeloid cells was most pronounced starting 2 weeks after the second cycle of chemotherapy, resulting in an optimal immunological 
window for vaccination [34]. Although these studies differ in tumor type, chemotherapy and type of vaccine, the most important difference could be the interval between chemotherapy and vaccination. In our study, this probably was too short to result in enhanced responses.

In this limited number of patients, we could not find a clear positive effect of the combination treatment on survival. A significant better OS is observed in stage III patients treated with combination therapy compared to DC monotherapy. However, groups are small and baseline characteristics too heterogenous to draw firm conclusions. For example, more stage IIIC patients were randomized in the DC monotherapy group. In addition, the difference in OS might be caused by ongoing responses to salvage therapy with immune checkpoint inhibitors (ICI) in two patients in the combination group while in the monotherapy group none responded to ICI. In addition, the clinical benefit was not supported by an increase in a clear increase in $\mathrm{T}$ cell responses. Finally, in stage IV, no significant difference in survival was found. Taken together, our data do not clearly suggest that addition of cisplatin to DC vaccination is of benefit to melanoma patients in the treatment schedule used.

On the other hand, cisplatin in combination with dexamethasone did not seem to harm immunological responses. More research is needed to optimize dosage and timing of cisplatin to assess its potential to enhance DC vaccination in vivo. Currently, ICI are available, and today, a study combining them with DC vaccination would, in melanoma patients, be preferred over combination with chemotherapy [35]. Combination might intensify proliferation and effector functions of tumor-specific T cells induced by DC vaccination by blocking inhibitory immune checkpoints with antiCTLA- 4 or anti-PD-1 mAbs [36, 37]. A recent phase 2 trial with DCs combined with anti-CTLA-4 mAbs showed tolerability and an encouraging objective response rate (38\%) in pre-treated advanced melanoma patients [38]. Further clinical trials, mainly with the less toxic and more effective anti-PD-1 mAbs, are under investigation, and results are awaited [39].

In conclusion, combination of autologous monocytederived DC vaccination and cisplatin in stage III and IV melanoma patients is feasible and safe, but enhancement of the tumor-specific $\mathrm{T}$ cell responses or clinical benefit when compared to DC monotherapy could not be confirmed in this limited number of patients. However, together with the currently available ICI, future research in melanoma patients should focus on the more promising combination of DC vaccination with ICI.

Acknowledgements The authors thank all patients for their interest and willingness to participate in the clinical trial. The authors thank the involved technicians of the Tumor Immunology Laboratory: Annemiek J. de Boer, Kevin J.H. Bos, Tjitske Duiveman-de Boer, Tom G.M. van Oorschot, Jeanette M. Pots, Nicole M. Scharenborg, Mandy W.M.M. van de Rakt, Michel A.M. Olde Nordkamp and Kiek N. Verrijp for their assistance with data collection. The authors thank Altuna Halilovic for analyzing the pSTAT3 expression.

Author contributions WJL and IJMdV were involved in conception and design of the trial. HW, SB and KFB managed the patients with supervision of CJAP, RHTK and WRG. MAJG and LLvdW performed immunohistochemistry experiments and analyses. $\mathrm{MB}, \mathrm{SB}$ and $\mathrm{NdH}$ collected and analyzed the data and wrote the manuscript under supervision of GS, KFB and IJMdV. All authors reviewed the manuscript.

Funding This work was supported by Dutch Cancer society grant KWF 2009-4402 and Vici grant 918.14.655 from the Netherlands Organization for Scientific Research.

\section{Compliance with ethical standards}

Conflict of interest Winald R. Gerritsen received speaker fees from Astellas, Bayer, Merck Sharp \& Dohme (MSD) and European Society for Medical Oncology, participated in advisory boards of Amgen, Bayer, Bristol-Myers Squibb (BMS), Curevac, Dendreon, IQVIA, Janssen-Cilag, MSD, Morphosys and Sanofi, and received research grants from Astellas, Amgen, Bayer, Janssen-Cilaq and Sanofi. Rutger H. T. Koornstra has received speaker fees from BMS, MSD and Roche. He has advisory relationships for BMS, MSD, Novartis and Roche. He received research grants from BMS and Roche. W. Joost Lesterhuis received research funding from Douglas Pharmaceuticals and Astra Zeneca. The authors declare that there are no other conflicts of interest.

Ethical approval and ethical standards All procedures performed in this trial were in concordance with the ethical standards of the institution and the 1964 Helsinki Declaration and its later amendments. The trial was approved by the Dutch Central Committee on Research Involving Human Subjects (study approval number: NL32381.000.10) and registered as NCT02285413 at ClinicalTrials.gov.

Informed consent Prior to inclusion, written informed consent to experimental treatment and analysis of data and tissue to answer research questions directly related to the trial was obtained from all patients.

Open Access This article is licensed under a Creative Commons Attribution 4.0 International License, which permits use, sharing, adaptation, distribution and reproduction in any medium or format, as long as you give appropriate credit to the original author(s) and the source, provide a link to the Creative Commons licence, and indicate if changes were made. The images or other third party material in this article are included in the article's Creative Commons licence, unless indicated otherwise in a credit line to the material. If material is not included in the article's Creative Commons licence and your intended use is not permitted by statutory regulation or exceeds the permitted use, you will need to obtain permission directly from the copyright holder. To view a copy of this licence, visit http://creativecommons.org/licenses/by/4.0/.

\section{References}

1. Boudewijns S (2017) Dendritic cell vaccination in the evolving therapeutic landscape of melanoma, departments of Tumor Immunology and Medical Oncology. Radboud University Medical Center, Nijmegen 
2. Bloemendal M (2019) Novel strategies in dendritic-cell based immunotherapy-focusing on adjuvant treatment of stage III melanoma, departments of Tumor Immunology and Medical Oncology. Radboud University Medical Center, Nijmegen

3. Palucka K, Banchereau J (2013) Dendritic-cell-based therapeutic cancer vaccines. Immunity 39(1):38-48

4. Boudewijns $\mathrm{S}$ et al (2016) Adjuvant dendritic cell vaccination induces tumor-specific immune responses in the majority of stage III melanoma patients. Oncoimmunology 5(7):e1191732

5. Aarntzen EH et al (2012) Vaccination with mRNA-electroporated dendritic cells induces robust tumor antigen-specific CD4+ and CD8+ T cells responses in stage III and IV melanoma patients. Clin Cancer Res 18(19):5460-5470

6. Lesterhuis WJ et al (2011) Route of administration modulates the induction of dendritic cell vaccine-induced antigen-specific $\mathrm{T}$ cells in advanced melanoma patients. Clin Cancer Res 17(17):5725-5735

7. Wilgenhof $\mathrm{S}$ et al (2011) Therapeutic vaccination with an autologous mRNA electroporated dendritic cell vaccine in patients with advanced melanoma. J Immunother 34(5):448-456

8. Bol KF et al (2016) Favorable overall survival in stage III melanoma patients after adjuvant dendritic cell vaccination. Oncoimmunology 5(1):e1057673

9. Dilruba S, Kalayda GV (2016) Platinum-based drugs: past, present and future. Cancer Chemother Pharmacol 77(6):1103-1124

10. Goodnight JE Jr et al (1979) Cis-dichlorodiammineplatinum(II) alone and combined with DTIC for treatment of disseminated malignant melanoma. Cancer Treat Rep 63(11-12):2005-2007

11. Luikart SD, Kennealey GT, Kirkwood JM (1984) Randomized phase III trial of vinblastine, bleomycin, and cis-dichlorodiammine-platinum versus dacarbazine in malignant melanoma. J Clin Oncol 2(3):164-168

12. Keilholz U et al (1997) Interferon alfa-2a and interleukin-2 with or without cisplatin in metastatic melanoma: a randomized trial of the European Organization for Research and Treatment of Cancer Melanoma Cooperative Group. J Clin Oncol 15(7):2579-2588

13. Bhatia S, Tykodi SS, Thompson JA (2009) Treatment of metastatic melanoma: an overview. Oncology (Williston Park) 23(6):488-496

14. de Biasi AR, Villena-Vargas J, Adusumilli PS (2014) Cisplatininduced antitumor immunomodulation: a review of preclinical and clinical evidence. Clin Cancer Res 20(21):5384-5391

15. Hato SV et al (2014) Molecular pathways: the immunogenic effects of platinum-based chemotherapeutics. Clin Cancer Res 20(11):2831-2837

16. Hato SV et al (2017) Direct inhibition of STAT signaling by platinum drugs contributes to their anti-cancer activity. Oncotarget 8(33):54434-54443

17. Wang $\mathrm{T}$ et al (2004) Regulation of the innate and adaptive immune responses by Stat-3 signaling in tumor cells. Nat Med 10(1):48-54

18. Lesterhuis WJ et al (2011) Platinum-based drugs disrupt STAT6mediated suppression of immune responses against cancer in humans and mice. J Clin Investig 121(8):3100-3108

19. Huang X, Cui S, Shu Y (2016) Cisplatin selectively downregulated the frequency and immunoinhibitory function of myeloidderived suppressor cells in a murine B16 melanoma model. Immunol Res 64(1):160-170

20. Roselli M et al (2013) Effects of conventional therapeutic interventions on the number and function of regulatory T cells. Oncoimmunology 2(10):e27025

21. Di Blasio S et al (2016) Human CD1c(+) DCs are critical cellular mediators of immune responses induced by immunogenic cell death. Oncoimmunology 5(8):e1192739
22. van der Sluis TC et al (2015) Vaccine-induced tumor necrosis factor-producing $\mathrm{T}$ cells synergize with cisplatin to promote tumor cell death. Clin Cancer Res 21(4):781-794

23. Balch CM et al (2009) Final version of 2009 AJCC melanoma staging and classification. J Clin Oncol 27(36):6199-6206

24. Finger PT (2009) The 7th edition AJCC staging system for eye cancer: an international language for ophthalmic oncology. Arch Pathol Lab Med 133(8):1197-1198

25. Berger TG et al (2002) Large-scale generation of mature monocyte-derived dendritic cells for clinical application in cell factories. J Immunol Methods 268(2):131-140

26. de Vries IJ et al (2005) Phenotypical and functional characterization of clinical-grade dendritic cells. Methods Mol Med 109:113-126

27. Schuurhuis DH et al (2009) In situ expression of tumor antigens by messenger RNA-electroporated dendritic cells in lymph nodes of melanoma patients. Cancer Res 69(7):2927-2934

28. Figdor CG et al (2004) Dendritic cell immunotherapy: mapping the way. Nat Med 10(5):475-480

29. Aarntzen EH et al (2012) Skin-test infiltrating lymphocytes early predict clinical outcome of dendritic cell-based vaccination in metastatic melanoma. Cancer Res 72(23):6102-6110

30. de Vries IJ et al (2005) Immunomonitoring tumor-specific T cells in delayed-type hypersensitivity skin biopsies after dendritic cell vaccination correlates with clinical outcome. J Clin Oncol 23(24):5779-5787

31. Van Nuffel AM et al (2012) Epitope and HLA-type independent monitoring of antigen-specific T-cells after treatment with dendritic cells presenting full-length tumor antigens. J Immunol Methods 377(1-2):23-36

32. Cook AM et al (2016) Dexamethasone co-medication in cancer patients undergoing chemotherapy causes substantial immunomodulatory effects with implications for chemo-immunotherapy strategies. Oncoimmunology 5(3):e1066062

33. Chen L, Jondal M, Yakimchuk K (2018) Regulatory effects of dexamethasone on NK and T cell immunity. Inflammopharmacology 26(5):1331-1338

34. Welters MJ et al (2016) Vaccination during myeloid cell depletion by cancer chemotherapy fosters robust $\mathrm{T}$ cell responses. Sci Transl Med 8(334):334ra52

35. Ribas A et al (2009) Dendritic cell vaccination combined with CTLA4 blockade in patients with metastatic melanoma. Clin Cancer Res 15(19):6267-6276

36. Tumeh PC et al (2014) PD-1 blockade induces responses by inhibiting adaptive immune resistance. Nature 515(7528):568-571

37. Morse MA, Lyerly HK (2015) Checkpoint blockade in combination with cancer vaccines. Vaccine 33(51):7377-7385

38. Wilgenhof S et al (2016) Phase II study of autologous monocytederived mRNA electroporated dendritic cells (TriMixDC-MEL) plus ipilimumab in patients with pretreated advanced melanoma. J Clin Oncol 34(12):1330-1338

39. van Willigen WW et al (2018) Dendritic cell cancer therapy: vaccinating the right patient at the right time. Front Immunol 9:2265

Publisher's Note Springer Nature remains neutral with regard to jurisdictional claims in published maps and institutional affiliations. 


\section{Affiliations}

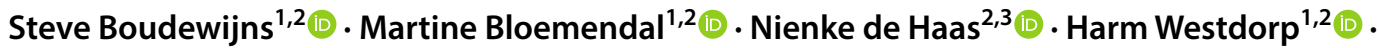

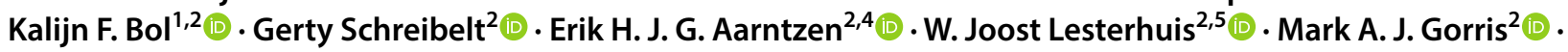
Alexandra Croockewit ${ }^{6}$ Lieke L. van der Woude ${ }^{2,7} \cdot$ Michelle M. van Rossum $^{8} \cdot$ Marieke Welzen $^{3} \cdot$ Anna de Goede $^{3}$. Stanleyson V. Hato ${ }^{2}$. Winette T. A. van der Graaf ${ }^{1}$. Cornelis J. A. Punt ${ }^{9}$ (1) - Rutger H. T. Koornstra ${ }^{1,10}$ (1)

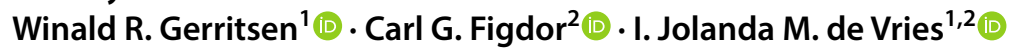

\section{Jolanda M. de Vries} jolanda.devries@radboudumc.nl

1 Department of Medical Oncology, Radboud University Medical Center, Nijmegen, The Netherlands

2 Department of Tumor Immunology, Radboud University Medical Center, Radboud Institute for Molecular Life Sciences, PO Box 9101, 6500 HB Nijmegen, The Netherlands

3 Department of Pharmacy, Radboud University Medical center, Nijmegen, The Netherlands

4 Department of Radiology and Nuclear Medicine, Radboud University Medical Center, Nijmegen, The Netherlands

5 School of Biomedical Sciences, University of Western Australia, Crawley, Australia
6 Department of Hematology, Radboud University Medical Center, Nijmegen, The Netherlands

7 Department of Pathology, Radboud University Medical Center, Nijmegen, The Netherlands

8 Department of Dermatology, Radboud University Medical Center, Nijmegen, The Netherlands

9 Department of Medical Oncology, Academic University Medical Center, University of Amsterdam, Amsterdam, The Netherlands

10 Oncological Center, Rijnstate Hospital, Arnhem, The Netherlands 